\title{
Strukturdynamik- und Maschinendiagnose an einem Cherenkov-Teleskop
}

\author{
Torsten Schneider, Robert Paeschke, Daniel Alarcón, Peter Blaschke*
}

\section{Zusammenfassung}

Das Deutsche Elektronen-Synchrotron (DESY) in Zeuthen entwickelt zurzeit die 12m-Teleskope im Rahmen des internationalen Cherenkov-Telescope-Array (CTA)-Konsortiums. Es ist geplant, die Teleskope über einen Zeitraum von mindestens 30 Jahren kostengünstig zu betreiben. Dabei führt der autarke Betrieb von Teleskopen an abgelegenen Standorten zu speziellen Anforderungen an die Ausfallsicherheit. Diese Anforderungen gelten sowohl für die Tragstruktur als auch für die Antriebskomponenten. Zustandsüberwachung und Verschleißanalysen sind in diesem Anwendungsfall mit klassischen Methoden der Maschinen- und Strukturüberwachung nur eingeschränkt möglich. Methoden der Mustererkennung aus dem Bereich der Psychoakustik konnten erfolgreich verwendet werden, um den Langsamlauf zu analysieren. In diesem Beitrag werden Ergebnisse von Schwingungsuntersuchungen an einem 1:1-Prototyp des Teleskops vorgestellt. Zusätzlich wird die Frage beantwortet, wie ein kostengünstiges Überwachungssystem mit minimaler Sensoranzahl realisiert werden kann.

\section{Abstract}

The Deutsche Elektronen-Synchrotron (DESY) in Zeuthen currently develops telescopes with $12 \mathrm{~m}$ diameter in the framework of the international Cherenkov Telescope Array (CTA) consortium. These telescopes are supposed to work cost-efficiently for a period of at least 30 years. Their operation at remote locations leads to special demands for the telescope's support structure and drive components system stability. In this case, traditional methods of condition monitoring and structural health monitoring reach their limits. Psychoacoustical methods could be successfully applied in order to analyse low revolution speed regime. In this paper, we present results of a structural dynamic analysis of a real-sized telescope prototype. Additionally, the question of how to realise a monitoring system with a minimum amount of sensors is addressed.

\section{EINLEITUNG}

Das Cherenkov Telescope Array (CTA) wird ein Verbund von ca. 100 optischen Teleskopen zur Analyse von astronomischen Gammateilchen (Actis et al. 2011). Für die CTA-Teleskope sieht die Planung einen kostengünstigen Betrieb über mindestens 30 Jahre an abgelegenen Standorten vor, wobei der Teleskopverschleiß die Messpräzision und die Ausfallsicherheit direkt beeinflusst (Schulz 2012, Schneider et al. 2014). So müssen z. B. Ausfälle des Antriebs während der Messungen unbedingt vermieden werden, damit immer eine Parkposition zum Schutz vor Wind und direkter Sonneneinstrahlung der empfindlichen Kamera erreicht wird.
Das CTA wird aus Teleskopen in drei verschiedenen Größen bestehen. Die mittelgroßen Teleskope mit $12 \mathrm{~m}$ Spiegeldurchmesser werden durch das Deutsche Elektronen-Synchrotron (DESY) in Zeuthen entwickelt. Abbildung 1, links, zeigt ein Foto des 2013 in Berlin-Adlershof errichteten 1:1-Prototyps, der dem Test des Konzepts und der Überprüfung der Spezifikationseinhaltung dient sowie das Sammeln von Erfahrungen beim Bau und Betrieb des Teleskops ermöglicht.

Aus schwingungstechnischer Sicht ist das Teleskop ein Hybrid aus Gebäude und Maschine, vergleiche Abbildung 1, rechts. Einerseits kann die (Trag-) Struktur wie ein Turm mit Stahlausleger schwingen, was zu ungewollten
Verschiebungen zwischen Kamera und Spiegeleinheit und somit zu Messungenauigkeiten oder schlimmstenfalls zu Schäden am Teleskop führen kann. Auf der anderen Seite enthält das Teleskop zur Ausrichtung mehrere Antriebe, deren Komponenten Maschinenschwingungen verursachen. Gemeinsam haben Gebäude- und Maschinenschwingungen, dass die Charaktere der Schwingungen Maßstäbe für den Zustand der Struktur bzw. des Antriebs enthalten. Diese Schwingungen sind damit ein günstiger, zerstörungsfreier Zugang zur Erfassung des jeweiligen Verschleißzustands. Abbildung 2 zeigt eine Einteilung der möglichen Analysetiefe. 
Zustandsüberwachung hat Alternativen, wie z. B. Fahren bis zum Bruch oder intervallbasierte Überwachung. Hauptvorteil der Zustandsüberwachung ist, dass zustandsorientierte Instandhaltung möglich wird, wodurch der Abnutzungsvorrat besser ausgeschöpft, unnötige Demontagen vermieden, beginnende Schäden frühzeitig erkannt und Folgeschäden weitgehend ausgeschlossen werden. Demgegenüber stehen als Hauptnachteil die Kosten für Planung, Sensorik, Messungen und Auswertungen.

Bei der Zustandsüberwachung wird zu Beginn ein Start-Zustand erfasst. Anschließend wird über einen längeren Zeitraum die Abweichung der Ist-Daten vom Start-Zustand beobachtet. Um Aussagen aus den Ist-Daten ableiten zu können (Zustandsdiagnose), sind zusätzliche Informationen aus Konstruktions- und Betriebsparametern notwendig (Theorie-Daten wie z.B. Drehzahl, Zahneingriffsverhältnisse und Schwingungsfrequenzen einzelner Getriebekomponenten). Die Zusammenführung von Konstruktions- und Betriebsparametern mit dem Neu- und Ist-Zustand ermöglicht die Zuordnung von Veränderungen dem Verschleiß einzelner Komponenten. Eine Zustandsprognose wird möglich, wenn Erfahrungen mit dem Verschleiß der einzelnen Komponenten vorliegen. Diese Erfahrungen können aus simulierten Schäden, aus einer Schadensdatenbank oder aus Expertenwissen stammen.

Nach den unterschiedlichen Anwendungsgebieten unterscheidet man das Condition Monitoring im Maschinenbau und das Structural Health Monitoring im Bauingenieurwesen (Kolerus 1995, Balageas et al. 2006). In diesem Beitrag wird eine ähnliche Zweiteilung genutzt. Im ersten Abschnitt werden die Besonderheiten und Einsatzmöglichkeiten von Maschinendiagnose für den Teleskop-Antrieb dargestellt. Der zweite Abschnitt erläutert den Einsatz und die Vorteile von Betriebsmodalanalysen zur Überwachung der Teleskopstruktur.

\section{UNTERSUCHUNG DES ANTRIEBS}

Das mittelgroße Cherenkov-Teleskop enthält zwei unterschiedliche Antriebe
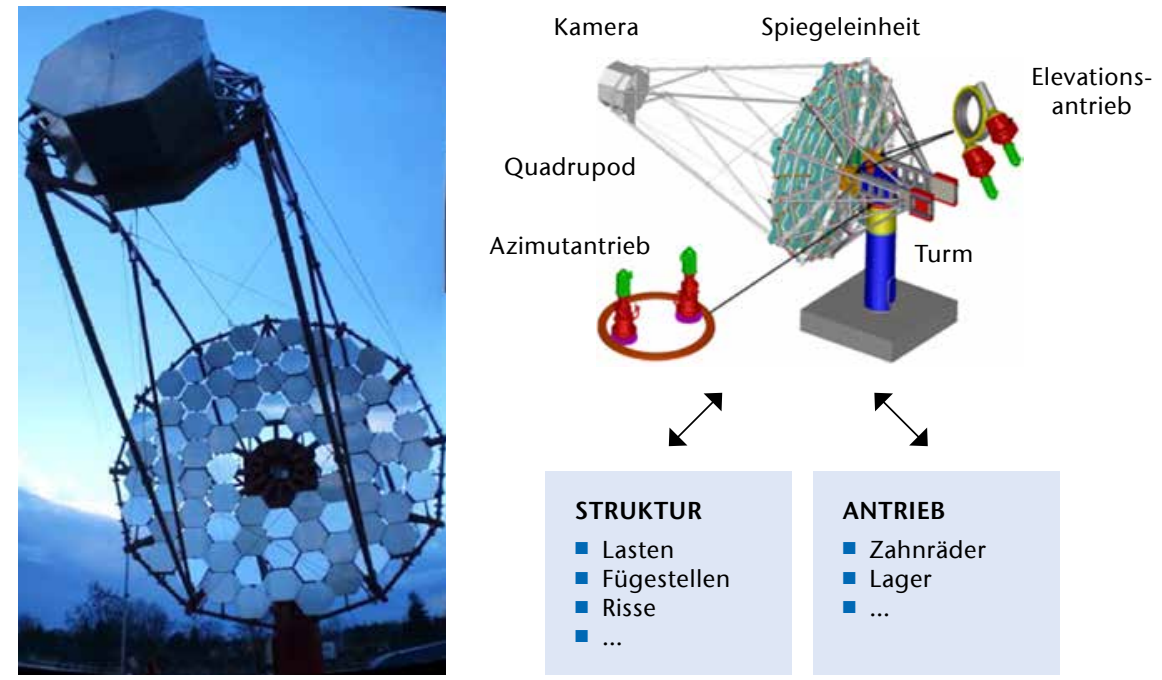

Abb. 1) links: Panoramafoto des Teleskop-Prototyps in Berlin-Adlershof vom Januar 2014. Rechts: Illustration des Teleskops und der schwingungstechnischen Aufteilung.

\section{ZUSTANDS-}

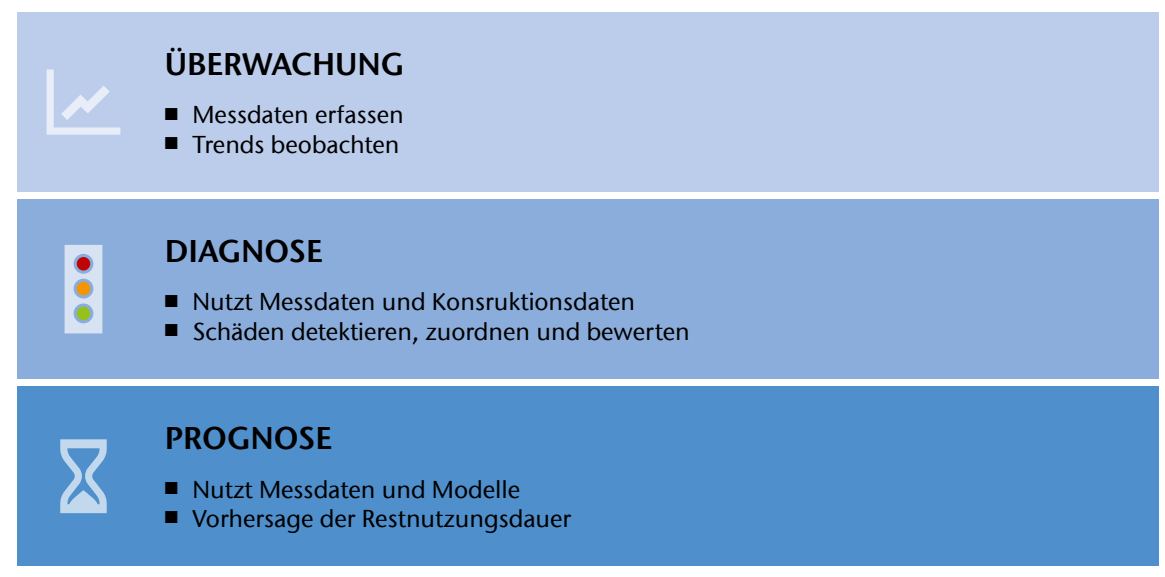

Abb. 2) Einteilung von Schwingungsanalysen im Bereich der Zustandsbeurteilung.

(vergleiche Abbildung 1, rechts): den Azimutantrieb für die Ausrichtung in horizontaler Richtung und den Elevationsantrieb für die vertikale Ausrichtung.

\subsection{MASCHINENDIAGNOSE}

Für die Maschinendiagnose werden Strukturschwingungen am Gehäuse der Antriebseinheit erfasst. Die Anregungsquelle der Strukturschwingungen sind die bewegten Lager und Zahnräder. Deshalb kann aus den gemessenen Schwingungsdaten auf die Anregungsquellen und deren Verschleiß bzw. Schäden zurückgeschlossen werden. Für den Stand der Technik im Bereich Maschinendiagnose kann z. B. das Nor- men- und Richtlinienwerk als Grundlage dienen (Gellermann \& Kolerus 2014). Obwohl es sich beim Teleskop nicht um Windenergieanlagen handelt, können aus diesem Bereich wertvolle Hinweise entnommen werden. Dies liegt daran, dass im Bereich der Windenergieanlagen nicht nur Maschinenschwingungsemissionen, sondern auch -immissionen berücksichtigt werden.

Im Vergleich zu Standardmaschinen und -zustandsüberwachungen liegt allerdings eine Besonderheit des Teleskops vor: der extreme Langsamlauf des Antriebs mit bis zu 0,002 $\mathrm{min}^{-1}$. Aus diesem Grund muss die Anwendbarkeit der Standardmethoden erst nachgewiesen werden. 
Da der Prototyp nahezu neu ist, sind typischerweise keine oder nur minimale Zahnrad- und Lagerschäden vorhanden. Die Herausforderung dieser Studie besteht in der Tatsache, dass noch keine Erfahrungsdaten vom Prototyp vorhanden sind. Es ist demnach unbekannt, ob Fehler vorliegen, ob sie gegebenenfalls detektierbar sind und mit welchen Diagnosemethoden sie nachgewiesen werden können.

\subsection{STUDIE ZUR ANTRIEBSÜBERWACHUNG}

Ziele dieser Machbarkeitsstudie sind a) der Nachweis, ob über die Messung von Strukturschwingungen mit Beschleunigungsaufnehmern einzelne Antriebskomponenten den Messdaten zugeordnet werden können (Zuordnung Ist- vs. Theorie-Daten); b) die Identifizierung geeigneter Messstellen.

\subsubsection{MATERIAL UND METHODEN}

\section{Auswahl der Messstellen}

Der Prototyp besitzt zwei Motor-Getriebe-Einheiten für die Azimutbewegung und vier Motor-Getriebe-Einheiten für die Elevationsbewegung. Für diese Machbarkeitsstudie wurde als Beispiel der Strang 1 des Azimutantriebs ausgewählt. Dies geschah aus zwei Gründen: a) bessere Zugänglichkeit und Montagemöglichkeit für die Sensoren; b) Übertragbarkeit der Ergebnisse auf den zweiten Azimutantriebsstrang und auf den Elevationsantrieb sind möglich.

\section{Messstellen}

Abbildung 3 zeigt die Bezeichnungen und die Sensorpositionen. Für die Messung der Strukturschwingung wurde der Antriebsstrang 1 mit piezoelektrischen Beschleunigungssensoren bestückt. Entsprechend der Unterteilung des Antriebsstrangs erfolgte jeweils die Montage eines Sensors. An jedes Gehäuse dieser Bauteile wurde mittig und radial von außen ein Sensor montiert. Die Beschleunigungssensoren wurden mit Parrafinwachs ablösbar an die Oberfläche geklebt.

\section{Messtechnik}

Aus den Theorie-Daten folgt, dass die höchste Frequenz des Azimutantriebs bei $1640 \mathrm{~Hz}$ liegt. Diese Frequenz

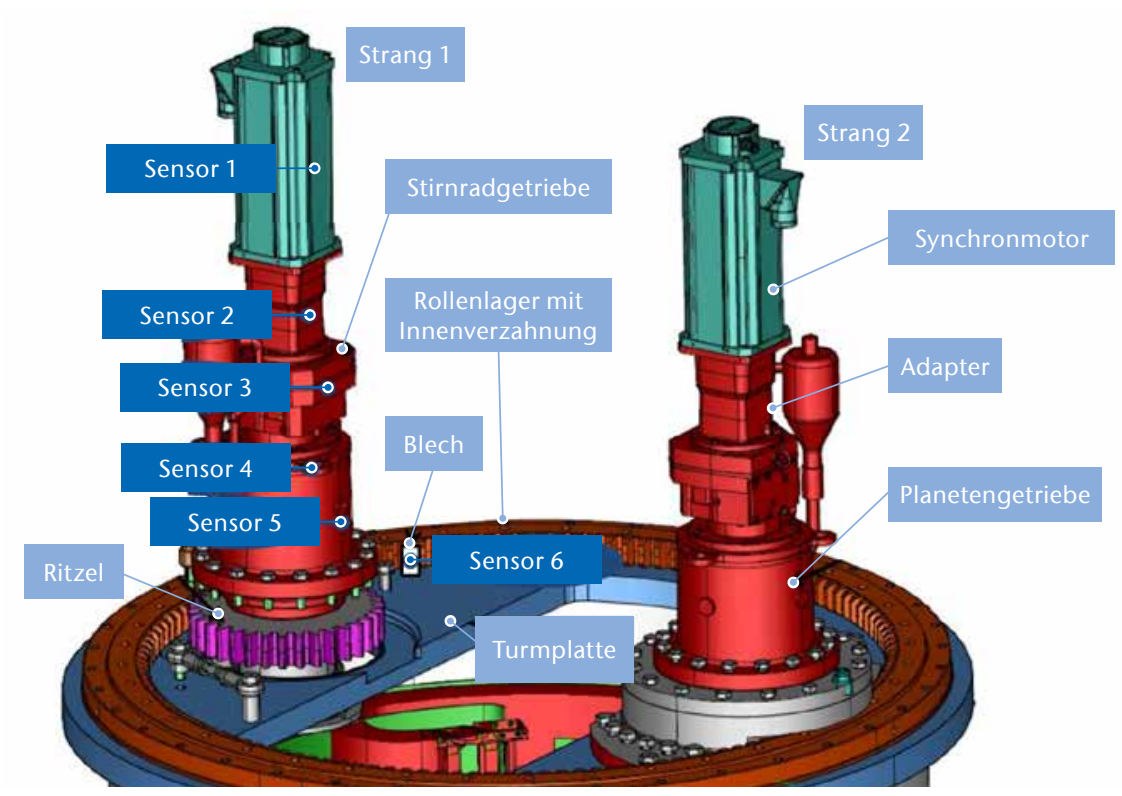

Abb. 3) Azimutantrieb mit Bezeichnung der Stränge, Antriebskomponenten und Sensorpositionen.

kann von der gesamten Messkette erfasst werden. Die Aufnahme der Sensordaten erfolgte über das mobile Datenerfassungssystem SQuadriga Il der Firma HEAD Acoustics (Herzogenrath, Deutschland) mit acht synchron abgetasteten Eingangskanälen mit einer Abtastrate von $32 \mathrm{kS} / \mathrm{s}$. Die Tiefpassfilterung für das Anti-Aliasing erfolgte intern im Gerät. Für die Schwingungsmessungen wurden piezoelektrische 1- und 3-AchsBeschleunigungssensoren der Firma PCB (PCB Piezotronics, NY, USA) verwendet.

\section{Messdurchführung}

In Abstimmung mit dem DESY wurde als repräsentative, reproduzierbare Betriebsbedingung folgende Konfiguration gewählt (im Folgenden: Diagnose-Fahrt): azimutale Drehung von $-269^{\circ}$ bis $+269^{\circ}$ mit Elevationswinkel $0^{\circ}$ bei maximaler Geschwindigkeit. Während der Diagnose-Fahrt wird das Teleskop beschleunigt, erreicht und hält die maximale Geschwindigkeit $\mathrm{v}_{\max }$ und wird anschließend wieder abgebremst. Während der Messung konnten die Antriebsdaten nicht synchron erfasst werden. Deshalb werden für diese Studie repräsentative Daten zur Analyse aus einer anderen Messung herangezogen. Die Drehzahl des Motors ist nach der Beschleunigungsphase nahezu konstant und beträgt 3942 $\pm 0,5 \mathrm{~min}^{-1}$ (Mittelwert \pm Standardabweichung).

\subsubsection{ERGEBNISSE UND DISKUSSION}

\section{Messwertevergleich}

Die gemessenen Beschleunigungen liegen als Zeitverläufe vor. Abbildung 4 zeigt eine beispielhafte Gegenüberstellung von verschiedenen Signalen.

In Abbildung 4 wird deutlich, dass während der Diagnose-Fahrt an allen Messstellen für Analysen ausreichende Signalamplituden nachgewiesen wurden. Dabei sind die Beschleunigungsamplituden an Motor, Adapter und Stirnradgetriebe ähnlich groß. Als ergänzende Testmessung wurde eine Aufnahme im Teleskopstillstand zur Bestimmung des Signal-Rausch-Verhältnisses durchgeführt: Die maximale Amplitude der gemessenen Hintergrundschwingungen beträgt ca. 0,4\% der Amplituden während der Diagnose-Fahrt bei $v_{\max }$ und ist damit vernachlässigbar klein. Um eine Auswahl für eine repräsentative Messstelle treffen zu können, wurde zusätzlich nachgewiesen, dass die gesuchten spektralen Anteile an allen Sensorpositionen enthalten waren.

\section{Musteranalysen}

Methoden der Mustererkennung aus dem Bereich der Psychoakustik konnten erfolgreich verwendet werden, um den Langsamlauf zu analysieren. Musteranalysen werden möglich, wenn die Frequenzanalyse zeitaufgelöst erfolgt. Muster werden dann 
in Zeitrichtung (z. B. Amplitudenmodulation), in Frequenzrichtung und kombiniert (komplexe Geräusche wie Quietschen) detektierbar. In den entstehenden Spektrogrammen ist in der Zeit-Frequenz-Ebene die Amplitude der einzelnen Frequenzen farbcodiert dargestellt. Abbildung 5 zeigt ein Beispielspektrogramm. Drehzahlunabhängige Schwingungsquellen lassen sich in den Beschleunigungsphasen anhand ihrer zeitachsenparallelen Frequenzverläufe klar von drehzahlabhängigen Quellen mit variablen Frequenzverläufen trennen. Drehzahlunabhängig sind am Teleskop beispielsweise die Grundfrequenzen $(4 \mathrm{kHz})$ der Motorelektronik und deren Harmonische. Drehzahlabhängige Schwingungsquellen sind $z$. B. die Zahnräder und die Lager des Antriebs. Deren Frequenzverläufe starten beim Stillstand von $\mathrm{OHz}$ und folgen der Motordrehzahl. Bei konstanter Fahrt mit $\mathrm{v}_{\max }$ beschreiben sie auch eine zeitachsenparallele Linie. Mit abnehmender Fahrt sinkt ihre Frequenz zurück auf die Nullinie. Amplitudenmodulationen werden als Änderungen in der Farbkodierung entlang der Frequenzverläufe erkennbar. In diesen Modulationen können auch sehr niedrige Frequenzen identifiziert werden. Dies gilt bis unterhalb der unteren Grenzfrequenz des Messsystems (hier ca. $5 \mathrm{~Hz}$, bedingt durch die Sensorbauart und die Frequenzauflösung der Analyse).

\section{Komponentenidentifikation}

Insgesamt enthält ein Antriebsstrang 31 Lager- und Zahnradkomponenten bzw. Schwingungsquellen. Dabei ist zu beachten, dass im Spektrogramm einer Diagnose-Fahrt weit mehr Frequenzen prominent erscheinen, als in den Theorie-Daten enthalten sind. Dies hat verschiedene Ursachen: a) Schwingungsquellen, die nicht zu den mechanischen Komponenten des Antriebs gehören (z.B. Frequenzumrichter); b) es treten aufgrund von Nichtlinearitäten auch Harmonische und Nebenbänder der theoretisch berechneten Frequenzen auf.

Ein Ziel dieser Studie war es, zu zeigen, in welchem Frequenzbereich Komponenten in den Messdaten identifiziert werden können, wobei der Betriebsfrequenzbereich der Antriebskom-
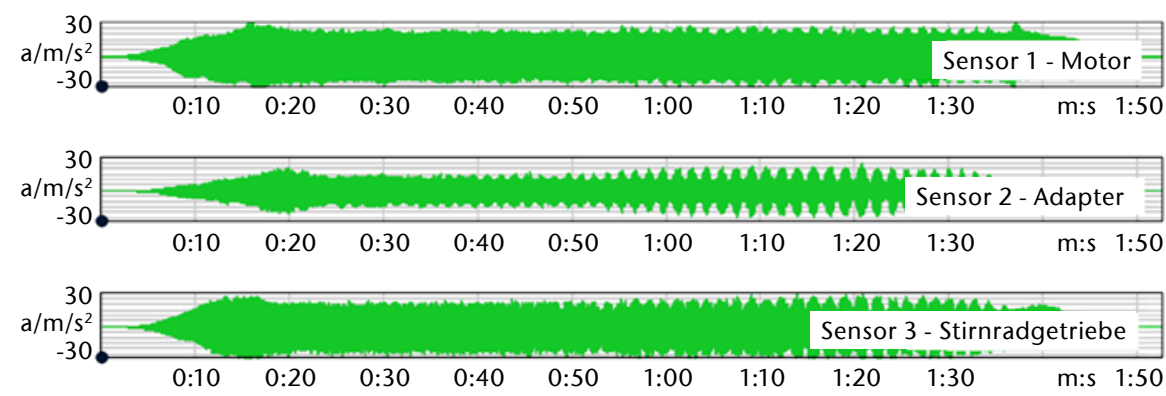

Abb. 4) Gegenüberstellung von gemessenen Zeitverläufen während der Diagnose-Fahrt an verschiedenen Sensorpositionen.

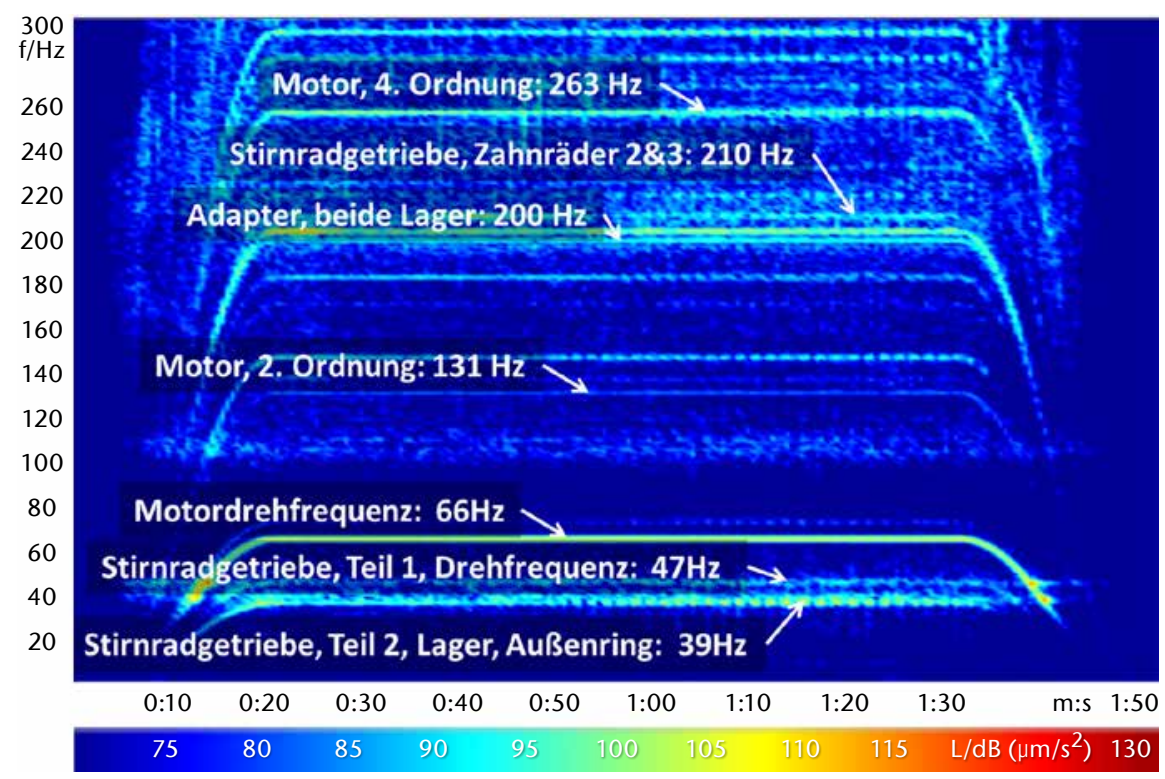

Abb. 5) Spektrogrammausschnitt (0 bis $300 \mathrm{~Hz}$ ) der Diagnose-Fahrt mit beispielhafter Zuordnung von Antriebskomponenten.

ponenten bei $v_{\max } z$ wischen $1640 \mathrm{~Hz}$ und $2 \mathrm{~Hz}$ liegt. Diese Identifizierung war im Bereich zwischen $1640 \mathrm{~Hz}$ und $39 \mathrm{~Hz}$ möglich. Damit können gegenwärtig die Komponenten des Motors und des Stirnradgetriebes identifiziert werden. Ursache für das Fehlen der motorfernen Komponenten ist deren Langsamlauf. Damit ist im ungeschädigten Komponentenzustand eine kleinere Quellenstärke verbunden. Es ist zu erwarten, dass bei beginnendem Verschleiß auch die Frequenzen der motorfernen Komponenten an Prominenz gewinnen.

\subsection{EMPFEHLUNGEN FÜR DEN CHERENKOV-TELESKOP-ANTRIEB}

\section{Empfehlung 1}

Weitere Messungen sollten für Trend- analysen durchgeführt werden. Die vorliegenden Analysen liefern einen Satz von Neudaten für zukünftige Diagnosen. Für die Entwicklung und die Implementierung eines CTA-Maschinendiagnosesystems ist eine möglichst vollständige Trendbeobachtung und umfangreiche Erfahrungsbasis notwendig. Diese kann nur auf kontinuierlich wiederholten Messungen mit frequenzaufgelösten Vergleichen über lange Betriebszeiten basieren. Stück für Stück wird eine Wissensdatenbank aufgebaut, die Ursachenidentifizierung sowie Festlegungen von Toleranzschemen, Warn- und Alarmwerten ermöglicht.

\section{Empfehlung 2}

Die Zustandsüberwachung einer Antriebseinheit (Motor + Getriebe) ist 
mit einem Beschleunigungssensor möglich. Die Analysen haben gezeigt, dass ein Beschleunigungssensor wegen der hohen Gehäusesteifigkeiten prinzipiell die Betriebsfrequenzen aller Antriebskomponenten erfassen kann. Wenn möglich sollte der eingesetzte Sensor speziell für den Betriebsfrequenzbereich von $2 \mathrm{~Hz}$ bis $1640 \mathrm{~Hz}$ angepasst sein.

\section{Empfehlung 3}

Es sollte ein Lastenheft für ein Maschinendiagnosesystem erstellt werden. Da nachgewiesen wurde, dass am Prototyp durch die Anwendung der Diagnose-Fahrt auswertbare Daten gemessen und bereitgestellt werden können, kann auf dieser Basis ein Lastenheft erstellt werden. Für die Erstellung sollte klar definiert werden, was die gewünschten Ziele des CTADiagnosesystems sind bzw. welche Möglichkeiten der Schwingungs- und Zustandsbeurteilung benötigt werden (Beurteilung der Schwingungen; Ampelsystem für Antriebszustand; zusätzliche Alarmanzeige für Spezialereignisse; Überlast; Verfolgung des Zustands als Trendkurven von Kennwerten; Grad der Auswerteautomatisierung etc.).

\section{Empfehlung 4}

Der Betriebszustand sollte bei Schwingungsmessungen mit erfasst werden. In der Antriebssteuerung liegen Daten bezüglich Drehzahl, Drehmoment, Drehrichtung und Ausrichtung vor. Für zukünftige Schwingungsmessungen sollten diese zusammen mit den Wetterdaten in die Datenerfassung integriert werden. Des Weiteren ist die Erfassung der Betriebszustände und -zeiten empfehlenswert, um die Zuordnung von Komponentenveränderungen zur Belastungshistorie möglich zu machen.

\section{UNTERSUCHUNG DER STRUKTUR}

Die Tragstruktur des Teleskops ist eine seilverspannte Stahlkonstruktion. Auf dem festen Turm ist der Ausleger beweglich gelagert. Die Hauptkomponenten des Auslegers sind die Spiegeleinheit mit 12 m Durchmesser, der Quadrupod mit einer Tiefe von ca. 20 m, die Gegengewichte und die ca. $2 \mathrm{t}$ schwere Kamera. Die Struktur muss neben statischen Lasten insbesondere Schwingungen durch dynamische Windlasten widerstehen.

\subsection{MÖGLICHKEITEN DER STRUKTURDIAGNOSE}

Sind für ein System die anregenden Kräfte und die Schwingungsantwort bekannt, kann aus deren Verhältnis auf die dynamischen Eigenschaften der Struktur in Form von Eigenfrequenzen, Eigenformen und modalen Dämpfungen geschlossen werden. Dieses klassische Verfahren ist als Struktur- oder speziell experimentelle Modalanalyse etabliert (Ewins 1984). Die kontinuierliche Kenntnis der modalen Eigenschaften einer Struktur ermöglicht die Zustandsüberwachung und -diagnose der Struktur. Dies ist möglich, da Verschleiß und Schäden die modalen Parameter z. B. über Steifigkeitsänderungen oder zusätzliche Fügestellendämpfung beeinflussen.

Bei großen Objekten und Gebäuden besteht allerdings das Anwendungsproblem, dass die anregenden Kräfte unbekannt sind und als Störgröße in die Analyse eingehen.

Eine Alternative zur klassischen Modalanalyse bietet die Betriebsmodalanalyse (Operational Modal Analysis, OMA). Die OMA ermöglicht über statistische Analysen von Schwingungsantworten (output only) die Bestimmung der modalen Eigenschaften, was die Anforderungen an Versuchsaufbau und -durchführung erheblich vereinfacht (Andersen 1997).

\subsection{STUDIE ZUR STRUKTURÜBERWACHUNG}

Zur Untersuchung, ob der TeleskopPrototyp die CTA-Spezifikationen erfüllt, hat das DESY ein Sensorsystem installiert. Dieses sollte für Voruntersuchungen genutzt werden, um die Frage zu beantworten, inwieweit diese Zeitreihendaten auch für die Durchführung von Betriebsmodalanalysen genutzt werden können.

\subsubsection{MATERIAL UND METHODEN}

Der Elevationswinkel des Teleskops war während der Messungen $0^{\circ}$ und die Schwingungsanregung erfolgte durch Wind. Es wurden insgesamt fünf zweiachsige, kapazitive Beschleunigungs-(Neigungs-)Sensoren des Typs KAS901-5X (Kelag, Schwerzenbach, Schweiz) eingesetzt. Die Abtastung erfolgte mit NI-9205-Analogmodulen (16 bit, Summenabtastrate $250 \mathrm{kS} / \mathrm{s}$ ) im NI cDAQ-9188 Chassis (National Instruments, Austin, Texas, USA). Die Sensoranordnung ist in Abbildung 6 und die Messkomponenten sind in Tabelle 1 dargestellt.

Die Messdaten werden mit einem digitalen Tiefpass bei einer Grenzfrequenz von $100 \mathrm{~Hz}$ gefiltert und als ca. $45 \mathrm{~min}$ lange Messschriebe gespeichert.

Zusätzlich wurden Finite-ElementSimulationen (FEM) der ersten Struktur-Eigenfrequenzen des TeleskopPrototyps durch das DESY berechnet. Ausgehend von diesen Daten der simulierten FEM-Moden wurden Frequenzen bis $10 \mathrm{~Hz}$ als interessanter Bereich ausgewählt.

Zur Durchführung der Betriebsmodalanalysen wurde die kommerzielle Software ARTeMIS Modal 2.5.0.5 (Structural Vibration Solutions A/S, Aalborg, Dänemark) eingesetzt. Die DESY-Daten wurden importiert und im Originalformat (in Volt) belassen, da dies keinen Einfluss auf die ermittelten modalen Größen (Frequenzen, Dämpfung, Formen) hat. Vor der Berechnung der modalen Parameter wurden die Daten auf eine Auflösung von $10 \mathrm{~Hz}$ reduziert (resampling). Zur Visualisierung der Eigenformen wurde eine vereinfachte Skelett-Geometrie der Messpunkte erstellt.

\subsubsection{ERGEBNISSE UND DISKUSSION}

Abbildung 7 zeigt die Singular Value Decomposition der Messungen, die einer Eigenwertzerlegung entspricht. Einfach gesagt: Die Spitzen in diesem Graphen könnten zu Eigenfrequenzen gehören. Abbildung 7 zeigt auch einige Eigenschaften der analysierten Daten: a) unter $2 \mathrm{~Hz}$ sind die Daten relativ stark verrauscht und $b$ ) für $1,135 \mathrm{~Hz}, 2,293 \mathrm{~Hz}, 2,637 \mathrm{~Hz}, 5,848 \mathrm{~Hz}$ und $6,877 \mathrm{~Hz}$ werden automatisch Kandidaten für Schwingungsmoden erkannt (Modal Domain). Zur Ermittlung der modalen Parameter wur- 
den EFDD-Auswertungen (Enhanced Frequency Domain Decomposition) durchgeführt (zu den Details dieser Methode siehe Jacobsen et al. (2006)). Die Ergebnisse sind in Tabelle 2 gelistet.

Die Eigenformen aus FEM-Simulationen und OMA passen sehr gut zusammen. Die Absolutwerte in Tabelle 2 zeigen allerdings bemerkenswerte Abweichungen zwischen simulierten und gemessenen Eigenfrequenzen. Abweichungen zwischen Simulation und Experiment sollten eigentlich im Bereich von $10 \%$ liegen. Ein Grund für die größeren Abweichungen könnte im FEM-Modell liegen. Dies ist aber kein Fehler der FEM, sondern resultiert aus notwendigen, unsicheren Annahmen. Erst wenn Kenntnisse von realen Messergebnissen vorliegen, kann die Unsicherheit der Annahmen reduziert werden. So sind FEM-Simulationen typischerweise zu steif (feste Verbindungen zwischen einzelnen Elementen), was in zu hohen Eigenfrequenzen resultiert. Auch die Modellierung von Getriebesträngen mit vielen einzelnen Zahnrad- und Wellensteifigkeiten erfordert fast immer eine Modellaktualisierung auf der Basis von Messwerten. Die Klärung dieser These erfordert eine Analyse und Aktualisierung des FEM-Modells.

Die ermittelten modalen Dämpfungen der ersten beiden Moden mit $7 \%$ und $11 \%$ sind relativ hoch. Für „normale“ Strukturschwingungen werden Dämpfungen im niedrigen einstelligen Prozentbereich erwartet. Allerdings müssen zwei Aspekte bei der Beurteilung berücksichtigt werden: a) die hohe Dämpfung könnte aus der Anbindung an die Getriebe und damit aus einer Kombination aus Struktur- und Getriebeschwingung stammen (1. Mode - Schwingung um Azimutantrieb; 2. Mode - Schwingung um Elevationsantrieb) und b) die ersten beiden Moden sind in Abbildung 7 nur verrauscht zu erkennen.

Zur Validierung der OMA-Ergebnisse kann das Modal Assurance Criterium als MAC-Matrix herangezogen werden. In Kürze: Die Einträge der MACMatrix geben an, ob die einzelnen Moden (wie theoretisch gewünscht)

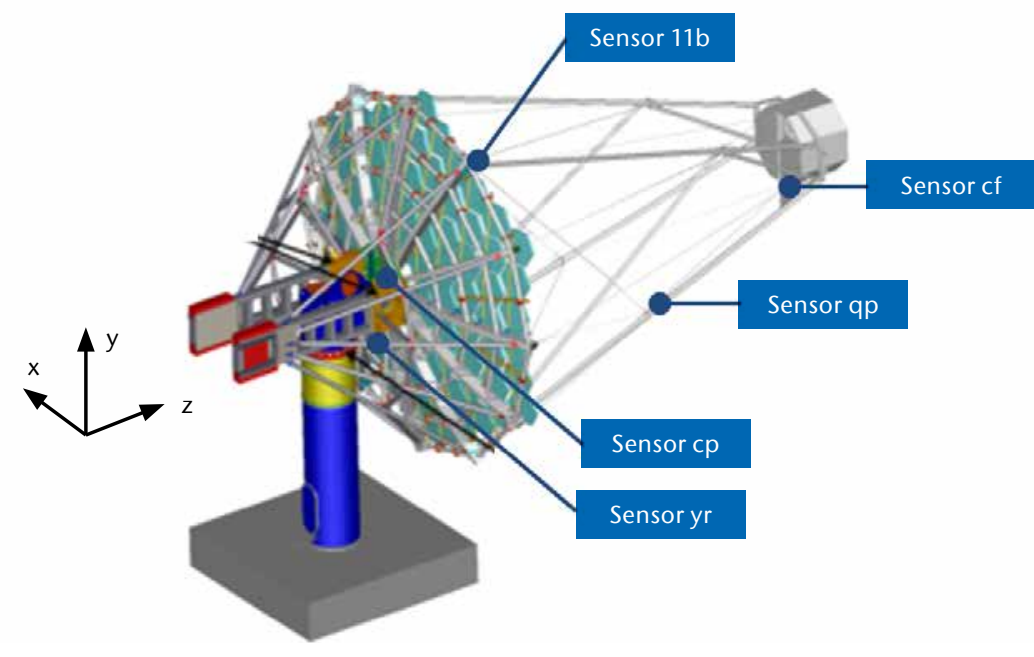

Abb. 6) DESY-Sensorsystem zur Erfassung von Strukturschwingungen.

Tab. 1: Übersicht zu Sensorpositionen am Teleskop-Prototyp (cf camera frame; qp quadrupod; 11b 11 o'clock beam; cp center plate; yr yoke right).

\begin{tabular}{|l|c|c|c|c|c|c|c|c|c|c|c|}
\hline Sensornr. & \multicolumn{1}{|c|}{1} & \multicolumn{2}{|c|}{2} & \multicolumn{2}{|c|}{3} & \multicolumn{2}{|c|}{4} & \multicolumn{2}{|c|}{5} \\
\hline Position & $\mathrm{cf}$ & $\mathrm{cf}$ & $\mathrm{qp}$ & $\mathrm{qp}$ & $11 \mathrm{~b}$ & $11 \mathrm{~b}$ & $\mathrm{cp}$ & $\mathrm{cp}$ & $\mathrm{yr}$ & $\mathrm{yr}$ \\
\hline Richtung & $-\mathrm{x}$ & $-\mathrm{y}$ & $\mathrm{x}$ & $-\mathrm{y}$ & $-\mathrm{x}$ & $-\mathrm{z}$ & $\mathrm{x}$ & $\mathrm{y}$ & $-\mathrm{z}$ & $-\mathrm{x}$ \\
\hline
\end{tabular}

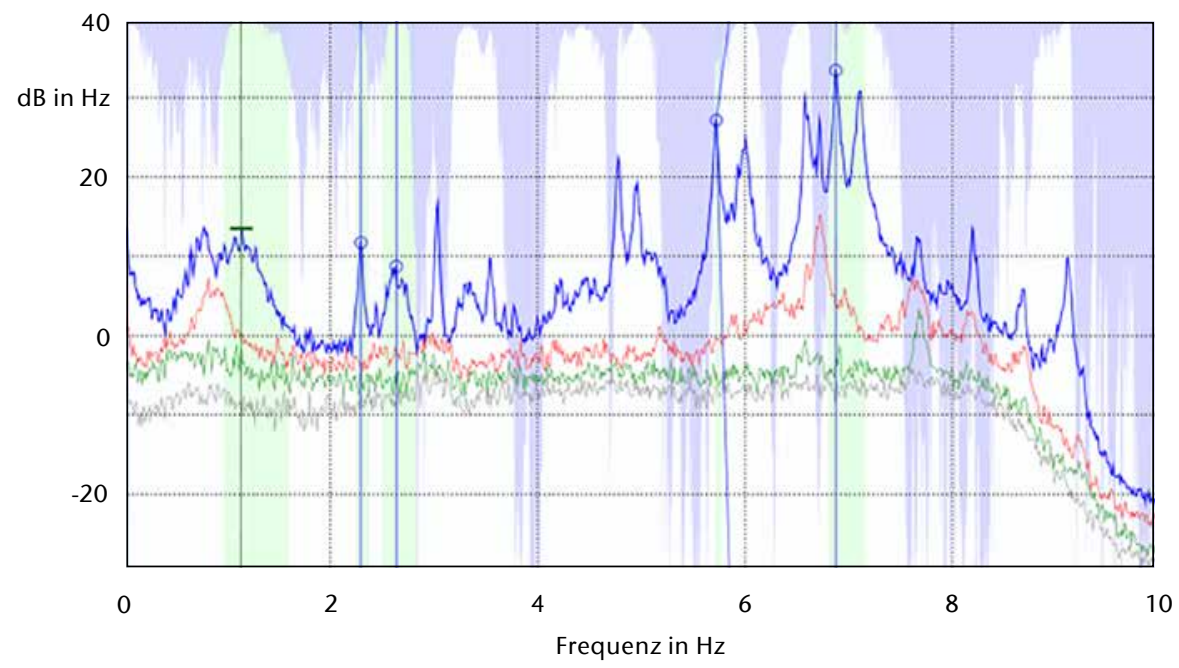

Abb. 7) Singular Value Decomposition der DESY-Daten zusammen mit automatisch detektierten Modalgebieten (grün).

Tab. 2: Gegenüberstellung von modalen Größen aus Finite-Element-Simulationen (FEM) und experimenteller Betriebsmodalanalyse (OMA). Nur im Experiment kann die Dämpfung bestimmt werden.

\begin{tabular}{|c|c|c|c|c|}
\hline Mode & Form & \multicolumn{2}{|c|}{ Eigenfrequenz in $\mathrm{Hz}$} & Dämpfung in \% \\
\hline & & FEM & OMA & OMA \\
\hline 1 & Teleskop-Kopf-Gieren & 1,549 & 0,778 & 6,917 \\
\hline 2 & Teleskop-Kopf-Nicken & 2,052 & 1,135 & 11,243 \\
\hline 3 & Teleskop-Kopf-Rollen & 2,765 & 2,293 & 0,975 \\
\hline
\end{tabular}


zueinander linear unabhängig sind. Dabei stehen Werte nahe Eins für lineare Abhängigkeit (erwartet nur für den Vergleich derselben Moden und damit für Einträge auf der Matrixdiagonalen) und nahe Null für lineare Unabhängigkeit (erwartet für alle anderen Matrixelemente). Abbildung 8 zeigt die MAC-Matrix der ermittelten Moden. Dieses Ergebnis bestätigt, dass die ermittelten Moden linear unabhängig sind und dass damit jeweils Eigenfrequenzen vorliegen.

\subsection{EMPFEHLUNGEN FÜR DIE CHERENKOV-TELESKOP-STRUKTUR}

\section{Empfehlung 1}

Es wird empfohlen, eine OMA mit ausreichend vielen Sensoren an ausgewählten Positionen durchzuführen. Mit dieser Untersuchung kann gezielt der Grundzustand des Prototyps charakterisiert, das FE-Modell aktualisiert und die notwendigen (wenigen) Schlüsselpositionen für Sensoren eines Strukturdiagnosesystems identifiziert werden.

\section{Empfehlung 2}

Zwischenzeitlich wird empfohlen, weiter Intervallmessungen mit dem vorhandenen Sensorsystem durchzuführen. Durch den Aufbau einer Messhistorie inklusive Grundzustand kann eine Datenbasis für ein Strukturdiagnose-Konzept und die Entwicklung von angepassten Trendanalysen bzw. Warnwerten erstellt werden. Dabei kann der Einfluss verschiedener Umgebungs-, Datenerfassungs- und Teleskop-Parameter (z.B. Temperatur, Windrichtung und -stärke, digitale Tiefpassfilterung, Elevationswinkel) auf die modalen Eigenschaften (Frequenzen, Dämpfungen) berücksichtigt werden.

\section{ZUSAMMENFASSUNG UND AUSBLICK}

Die in dieser Studie präsentierten Ergebnisse von Schwingungsuntersuchungen stellen den momentanen Zustand des Antriebs und der Struktur des Cherenkov-Teleskop-Prototyps dar. Die Anwendbarkeit der Zustandsüberwachungsmethoden wurde in beiden Anwendungsfällen erfolgreich nachgewiesen: Wichtige Antriebskomponenten konnten in den Maschinenschwingungsdaten identifiziert und die

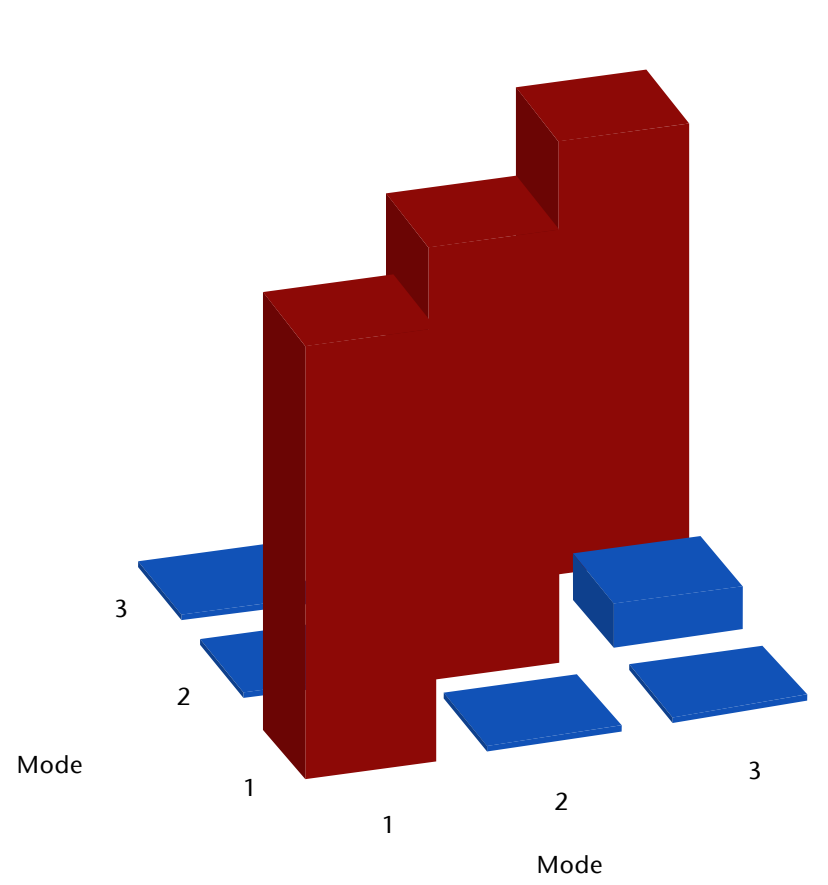

Farbzuordnung

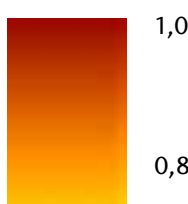

1,0

0,6

0,4

0,2

0,0

Abb. 8) Matrix des Modal Assurance Criterium (MAC) zu den ersten drei Moden aus der EFDD-Analyse.

modalen Parameter der Tragstruktur ermittelt werden. Es wurde gezeigt, wie es durch die Wahl von geeigneten Messpositionen und -bedingungen möglich ist, die Sensoranzahl zu minimieren. Damit reduziert sich der Aufwand für die Überwachung auf wenige Sensoren, was ein Vorteil für die serienmäßige Installation eines CTA-Teleskop-Diagnosesystems ist.

Allerdings wurden auch die Grenzen der durchgeführten Messungen aufgezeigt. Für die Diagnose von Antriebskomponenten muss die zugehörige Anregungsamplitude ausreichen bzw. der Sensormessbereich abgestimmt sein. Um den Einsatzbereich der Methode auszuloten, ist geplant, Schwingungen an einer Antriebseinheit mit künstlich eingefügten Lagerschäden zu vermessen. Die Strukturdiagnose benötigt lange Messdatenreihen, eine angepasste Datenerfassung und die Durchführung einer genaueren Betriebsmodalanalyse/OMA mit einem dichten Netz von angepassten Sensoren.

Der nächste Schritt liegt in der Erstellung einer Messhistorie durch kontinuierlich fortgesetzte Langzeitmessungen und Trendanalysen. Diese Datenbasis ist einerseits notwendig, um Grenzwerte für ein Teleskop-Dia- gnosesystem festlegen zu können und um andererseits Wissen für die weitere Teleskop-Entwicklung zu sammeln.

\section{DANKSAGUNG}

Diese Studie wurde aus Mitteln des Europäischen Sozialfonds und des Ministeriums für Wissenschaft, Forschung und Kultur des Landes Brandenburg finanziert (Projektnr. 1392533). Zusätzlich danken die Autoren A. Schubert, J. Schultze und R. Sternberger vom DESY-Zeuthen für die Unterstützung während der Messungen. 


\section{LITERATUR}

Actis M et al. (2011) Design concepts for the Cherenkov Telescope Array CTA: an advanced facility for groundbased high-energy gamma-ray astronomy. Exp Astron 32(3):193-316. doi: 10.1007/s10686-011-9247-0

Andersen P (1997) Identification of Civil Engineering Structures using Vector ARMA Models. PhD Thesis, Aalborg University

Balageas D, Güemes A, Fritzen C (eds) (2006) Structural health monitoring. ISTE, London, Newport Beach, CA. ISBN: 978-1-905209-01-9. doi: 10.1002/9780470612071

Ewins DJ (1984) Modal Testing: Theory and Practice. Mechanical engineering research studies, Engineering dynamics series, vol 2. Research Studies Press/Wiley, Letchworth, New York

Gellermann T, Kolerus I (2014) Schwingungsbeurteilung und Diagnostik von Windenergieanlagen im Spiegel von Normen und Richtlinien. In: Sauer B (ed) 5 . VDI-Fachtagung Schwingungen von Windenergieanlagen. VDI-Verlag, Düsseldorf, ISBN: 978-3-18-092220-1

Jacobsen N, Andersen P, Brincker R (2006) Using Enhanced Frequency Domain Decomposition as a Robust Technique to Harmonic Excitation in Operational Modal Analysis. In: Proceedings of ISMA2006: International Conference on Noise and Vibration Engineering, pp $18-20$

Kolerus I (1995) Zustandsüberwachung von Maschinen, 2nd edn. Kontakt \& Studium, 187: Maschinenbau. Expert-Verlag, Renningen-Malmsheim. ISBN: 978-3 8169-1168-5

Schneider T, Paeschke R, Alarcón D, Schulz S, Coswig D, Blaschke P (2014) Zustandsüberwachung von Teleskopen durch Schwingungsanalysen. In: Hochschule Magdeburg-Stendal (ed) 15. Nachwuchswissenschaftlerkonferenz, 24 Apr 2014, Magdeburg

Schulz S (2012) Erstellung eines Konzeptes zur strukturellen Zustandsüberwachung an Teleskopkomponenten. Bachelorarbeit, Technische Hochschule Wildau

\section{AUTOREN}

Dr.-Ing. Torsten Schneider Technische Hochschule Wildau Nachwuchsforschergruppe für strukturdynamische Optimierung torsten.schneider@th-wildau.de

Dipl.-Ing. Robert Paeschke

Technische Hochschule Wildau Nachwuchsforschergruppe für strukturdynamische Optimierung robert.paeschke@th-wildau.de

\section{B. Eng. Daniel Alarcón}

Technische Hochschule Wildau Nachwuchsforschergruppe für strukturdynamische Optimierung daniel.alarcon@th-wildau.de

Prof. Dr. Peter Blaschke

Technische Hochschule Wildau Professur für Maschinendynamik,

Konstruktion und CAD

peter-g.blaschke@th-wildau.de

http://www.th-wildau.de/maschinendynamik 